\title{
Applications of Wireless Sensor Networks
}

\author{
João M. Ferro, Luís M. Borges, Fernando J Velez, Member, IAENG, and António S. Lebres
}

\begin{abstract}
Wireless Sensor Networks (WSNs) are spreading around in multiple fields of applications because they perform measuring tasks in a flexible way. This paper presents a survey on nowadays research efforts on the application of these WSNs to the automobile industry, to agriculture, and to wearable technologies (healthcare and athletes monitoring). From our practical deployments, it is important to highlight the functionalities for the automotive industry, and the results extracted from our application to a vineyard. Research on wearable technologies constitutes a challenge, and wireless healthcare solutions in the context of fabrics are being addressed.
\end{abstract}

Index Terms - Agriculture applications, automotive applications, wearable technology, wireless sensor networks.

\section{INTRODUCTION}

WSN is a term used to describe an emerging class of embedded communication products that provide redundant, fault-tolerant wireless connections between sensors, actuators, and controllers. The large amount of research projects in this area allows for the existence of better tiny hardware devices with reduced cost/size, and improvements in software performance. WSNs are typically formed by groups of several sensor nodes, the so-called motes, whose individual constitution is based on actually combining sensor radios and CPUs into an effective robust secure and flexible network, with low power consumption, and advanced communication and computation capabilities, one or more sensors, a communication device (typically a radio), a microcontroller (with memory), and a power supply (battery), Fig. 1. These motes exchange messages among each other in order to efficiently monitor an environment/process.

The power of wireless sensor networks lies in the ability to deploy large numbers of tiny wireless nodes that assemble and configure themselves.

Manuscript received March 22, 2007, reveised April 16, 2007

J. M. Ferro, L. M. Borges, and F. J. Velez are with the Instituto de Telecomunicações, University of Beira Interior, Covilhã, Portugal (corresponding author to provide phone: +351 275329953; fax: +351 275329972; e-mail: joaomferro@gmail.com, luismiguelborges@ gmail.com, fjv@ubi.pt).

A. S. Lebres is with the Department of Physics (UDR), University of Beira Interior, Covilhã, Portugal (phone: +351 275319840; fax: +351 275319719; e-mail: lebres@ubi.pt).

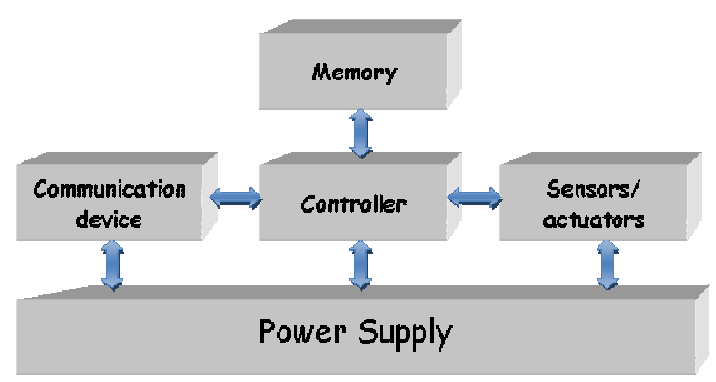

Figure 1 - Main sensor node hardware components

WSNs started to be developed for military use, aiming to have real-time information about the condition and distribution of the troops as well as the location of the enemies. However, in recent years their field of application has been well expanded, and WSNs are being used for real-time tracking, in industry to monitor processes (e.g., temperature and pressure inside ovens), in habitats [1] (where the species, animals or plants, can be observed without being disturbed by human presence), within environments (detection of fires [2], measurement of temperature, pressure and wind speed, etc.), to in situ monitoring of the condition of structures and equipment, in smart spaces (such as cars), and in medicine to monitor patients [3], [4], among others.

WSNs have specific characteristics different from common networks. One of the characteristics is the typical centralization of data, i.e., in the WSN the sensor nodes must take care of the specific requirements of the application. The nodes are focused in only one measure/attribute or in a small set of measures/attributes that need to be processed inside the network. The tolerance to the appearance of failures (very likely due to the fact that the sensors nodes are low cost) is another one of the characteristics of the WSN. The errors can occur, for example, due the energy failure, lack of communication, or lack of stability in software. Another important characteristic of WSNs is the scalability. As the sensor nodes are low cost and reduced size, it is possible to build up dense networks with a high number of nodes. The low consumption of energy is another basic characteristic of the system; hence, there are limitations to power supply in sensor nodes. Furthermore, motes must cooperate among them in order to efficiently exchange data, i.e., by using multi-hop to save energy. Besides, at the level of each individual sensor node, an efficient management of transmission, reception, and sleep modes of operation that should be implemented. Sensor nodes must remain in the sleep-mode whenever they do not have to receive/process/transmit data packets. 
This work presents a survey on applications for wireless sensor networks and addresses three practical deployments. Section II presents a brief survey on the use of WSNs in the automobile industry, precision agriculture and wearable technologies. Section III is dedicated to the application of WSNs to the automobile and covers routing and energy consumption aspects, and hardware components. Section IV is dedicated to precision agriculture, and presents the InterNodal network, with its sensor and master nodes. The initial practical results finally are discussed. Wearable technology devices are very useful in the fields of healthcare and athletes competition monitoring. Section V addresses recent developments in this area and gives an overview of possible research directions. Finally, conclusions are presented in Section VI.

\section{APPLICATIONS AND CHALLENGES}

The application of WSNs to the automobile constitutes a challenge and is faced as an endeavour. Perhaps the most welcomed innovation allowed by wireless sensors is the ability to monitoring tire pressure. Tire Pressure Monitoring (TPM) systems are becoming standard in the automobile industry and the TREAD Act [5] demands that every new light vehicle manufactured after 1/9/2007 in USA must include this system in all its wheels. Other types of applications will be based on the cooperative communication among cars to improve road safety, and prevent car accidents.

In another field, there are several ongoing projects using wearable devices/smart textiles. Sensatex launched the beta version of its SmartShirt in 2006, and interesting commercial results are being achieved. It monitors movement, hearth rate, and breathing rate by using nanotechnology-based fibres [6]. Several similar projects, such as the VATMN (Vêtement de Télé Assistance Médicale Nomade) [7] and the WEALTHY (Wearable Health Care System) [8] have produced functional prototypes. The Proetex project [9] is developing a wearable device that monitors health condition, activity and geographic position of emergency disaster crew (e.g., fire-fighters and civil protection workers). It will also support monitoring of the environment nearby to warn the wearer or somebody else of any risky situation (e.g., presence of toxic gases). Signalife announced in 2006 a plan to hook up its Fidelity 100 ECG to athletes [10]. A demonstration was carried out later that year with a cooperation of a known athlete, whose vital signals were monitored (and displayed in real-time) by 12 ECG leads

A WSNs prototype is being developed by UbiSec\&Sens to evaluate the suitability of security and reliability concepts for agriculture applications. Special components are being incorporated for a distributed storage of monitored data [11]. The Lofar Agro project is aimed for precision agriculture. This project concentrates on monitoring micro-climates (humidity and temperature) in crop fields and gathers statistics on the wireless sensor network that will be used for later prediction of the growing of crops [12]. In 2005, Grape Networks, Inc. announced that Napa Valley's Beringer Vineyards was implementing what was believed to be the first commercial wireless sensor network in a production vineyard. Small battery powered nodes were placed in the vineyard field to improve the quality of crops by communicating temperature, light information and moisture. All this data can be monitored anywhere in the world via the Internet [13]. Sandia National Labs is conducting research on the practicality and water savings potential of growing livestock forage in arid regions. The project was specifically focused on investigating the operational performance of a passive and relatively low-technology plastic covered greenhouse system for growing livestock forage hydroponically with significantly reduced water use in draught stricken areas [14].

\section{APPLICATION TO THE AUTOMOBILE INDUSTRY}

\section{A. Introduction}

The use of WSNs can be seen from two different points of view. Firstly, WSNs can form a network to interconnect several sensors inside the car. These networks aim to collect data about the conditions of the vehicle (and in some cases, of the driver), and provide it to the user or to an automatic control system. Secondly, WSNs can be formed by nodes which are cars themselves. This network is built very dynamically and the participants are the cars that are nearby. It is aiming to provide information about several situations, such has: i) the existence of others cars in the road, and their relative position, ii) the existence of pedestrians crossing the road, iii) accidents, or iv) traffic congestion [15].

As an initial experiment, we conceived a wireless sensor system capable to collect, process, and supply several types of technical information (to the user) during an automobile journey. Examples are acceleration and fuel consumption, identification of wrong tires pressure value, acknowledgment of illumination failures, and determination of the vital signs of the driver. We chose Crossbow MICAz sensors operating at $2.4 \mathrm{GHz}$ (IEEE 802.15.4), and supported by TinyOS. In this Section, the concepts of the wireless sensor network itself (transmitter/ receiver/ interface board) are explained, and aspects of the architecture and power consumption are discussed.

\section{B. Routing and energy consumption aspects}

There are several protocols in the context of TinyOS. As an example, the TinyOS Beaconing is a routing protocol used in Mica Motes wireless sensor nodes of the University of Berkeley, and operates within networks with restricted hardware [16]. The protocol periodically builds the Minimum Spanning Tree starting from the Base Station. The Base Station propagates the message (beacon call) that is spread through the network with the objective of creating the routing tree. As it is a simple and general protocol, its performance is lower than the one of protocols developed for specific applications.

From the essential hardware components needed, the transmitter is the largest energy consumer. Even when it is in the sleeping or idle modes, the transmitter wastes a considerable amount of energy. As so, it is very important to put the radio into sleep mode as much as possible, by using an appropriate 
energy-saving scheme. The node itself, when there are no readings to be done nor data to process, must also be put into a sleep mode.

\section{Hardware Components}

For the transmission and processing of the signals we have selected the MICAz ZigBee (MPR2400) from Crossbow, which has a range of 100 meters in line-of-sight $(\mathrm{LoS})$ and a range of 30 meters within walls. This module uses an ATMega 128L in order to collect the data from the network, and to program MICAz motes. The MIB510 interface board was connected to a computer by using the serial port, and the MTS310 sensor board was attached to the MICAz node.

Flow sensor - We chose a flow sensor with the technical, economical, and weight specifications closest to the requirements. We had to use a frequency to voltage converter, in order to have an appropriate voltage signal at the ADC input.

Tire pressure sensor - The pressure sensor included in tire pressure reader Sensor Monza 2 in 1 was the chosen one. The sensor has four terminals, which indicates that its internal circuit should be a Wheatstone bridge. To monitor the pressure value we use TinyDB, and the sensor node executes the TinyDBApp program.

Light Sensor - We opted for the light sensor already included into the MTS310 sensorial board, Fig. 2. To receive data from this sensor, the mote is programmed with OscilloscopRF application, while being placed onto the zone to be monitored. Another node, running the TosBase application, is placed on the interface board connected to the computer. Then, one can lively watch the variation of the light brightness on the photo-voltaic sensor of the mote, Fig. 2, and as so know how that lamp is.

Acceleration sensor - The acceleration sensor is incorporated into the MTS310 board of sensors. The signal acquisition is performed by using TinyDB.

Temperature sensor - The temperature sensor should be installed in contact with the driver's body in order to monitor his/her temperature. As the sensorial board already has a built-in temperature sensor, this sensor serves our initial objectives. The data of this sensor is read by TinyDB.

Heart frequency and arterial pressure - The equipment selected to measure the heart frequency and the blood pressure (diastolic and systolic) is the prototype for testing and monitoring the biomedical signals developed in the Department of Computer Science of University of Beira Interior. All the measuring procedure was effectively controlled by two dedicated nodes.

In our work, TinyDB was used for reading data from external sensors attached to the sensorial board, e.g., from the flow meter, the tire pressure sensor, and the blood pressure sensor. In these cases, we used the Digi-Key H2163-ND connector. In this work, the external sensors were connected to terminals 42 and 51 (Ground), corresponding to the light sensor in the sensorial board.

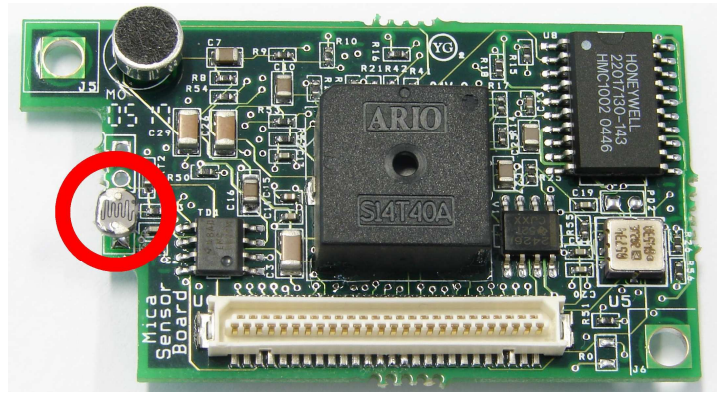

Figure 2 - Placement of the light sensor on the MTS310

\section{Summary and conclusions}

The conception of a WSN capable of measuring, processing and supplying different types of information about automobile behaviour was addressed. Examples were acceleration and fuel consumption, identification of incorrect tire pressure, verification of illumination, and evaluation of the vital signs of the driver. Besides a survey on the concepts, the wireless sensor network itself (transmitter/receiver/control board) was developed, and aspects of the architecture and protocols were addressed. Security aspects were also identified and the difficulties and implementation solutions were discussed. Competition cars in a controlled environment is a good scenario for experimental work, and the evolutions in this field promise a lot in the automobile industry, e.g., cooperation among cars for road safety purposes.

\section{PRECISION AgRiCUlture}

\section{A. InterNodal Network}

This Section addresses the integration of WSNs technologies in agriculture. The main objective is the application of WSNs to manage and monitor several physical parameters in an agricultural facility. The fundamental characteristics of our network, the so-called InterNodal Network [17], [18], are versatility, portability, self-configurability, and bi-directionality. A Master node is responsible for the coordination of the entire network and the establishment of the interface with a Personal Computer (PC). All the acquisition sensor nodes have data processing capabilities and may be expanded indefinitely through expansion ports. One important characteristic of this wireless network, is the data bi-directionality, i.e., the source nodes have the ability to collect all values from the sensors, and to send them, in real-time, to the Master node, as well as to receive data or commands from it. The InterNodal Network has a Master node and one or several Data Acquisition Processor/ Radio (DAPR) nodes, i.e. the portable nodes. DAPR nodes collect the physical data environment values and retrieve them to the Master node. Communication can be single-hop or multi-hop. In the latter case other DAPR nodes are used as relays. Therefore, DAPR nodes also receive and process information sent by the Master node. The main characteristics of DAPR nodes are the following: i) low stead state energy consumption, ii) 10 pre-settable transmission frequencies, iii) maximum range of 
approximately of 250 meters in line-of-sight (LoS), iv) high data processing capacity with reduced speeds of clock, vi) possibility of expansion, vii) capacity to change the channel of transmission, viii) control the transmission power level. The sensor node contains a microcontroller, a radio module (low power and short range) and a pin connector for the JTAG programming. The selected radio modules for this application are short range devices from Low Power Radio Solutions (LPRS) that operate at the $433 \mathrm{MHz}$ Industrial, Scientific, and Medical (ISM) band. The selected microcontroller to be inserted in DAPR nodes is a Texas Instruments MSP430P-1232, which has low power supply requirements, considering its actual clock frequency.

\section{B. References}

The Master node is used as a base station that coordinates the entire Internodal network and enables the interface with a computer through, e.g., a USB connection. The system administrator can operate and control the network via Ethernet, Internet or through Wi-Fi networks. The Master node used in this application contains an USB-serial converter, and a transceiver similar to those used in DAPR nodes, Fig. 3.

The InterNodal program was developed in $\mathrm{C}++$ with the purpose to control the network. With this interface we can observe in real-time the data values received from the sensors connected to DAPR nodes, as well as save the values of the collected data. Besides, the programme specification includes many other functions implemented, or susceptible to be implemented, according to the user application. A particular characteristic of this program is the possibility of sending the information of any DAPR node, usually urgent alarms, to the agricultural facility supervisor through SMS messages (Short Message Service).

The InterNodal network basic principle stands on the previous establishment of paths between nodes. During the InterNodal network configuration procedure, in an automatic way, each node is identified by a particular address, consisting of two bytes. The first byte is called "Dominant Address" and is the same for all the devices in the same network. The second byte must be unique for each node, and must identify individually each node in the InterNodal network. This $2^{\text {nd }}$ byte of address can have values between 1 and 63 , being the $64^{\text {th }}$ value reserved for the Master node.

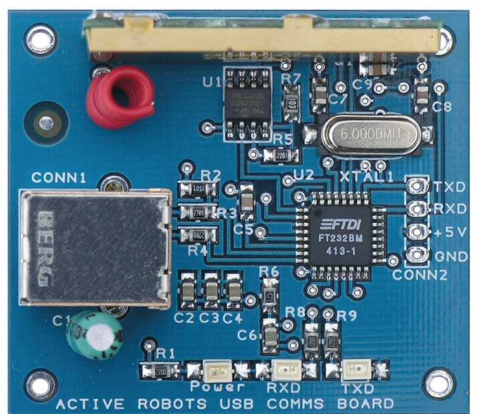

Figure 3 - Master node

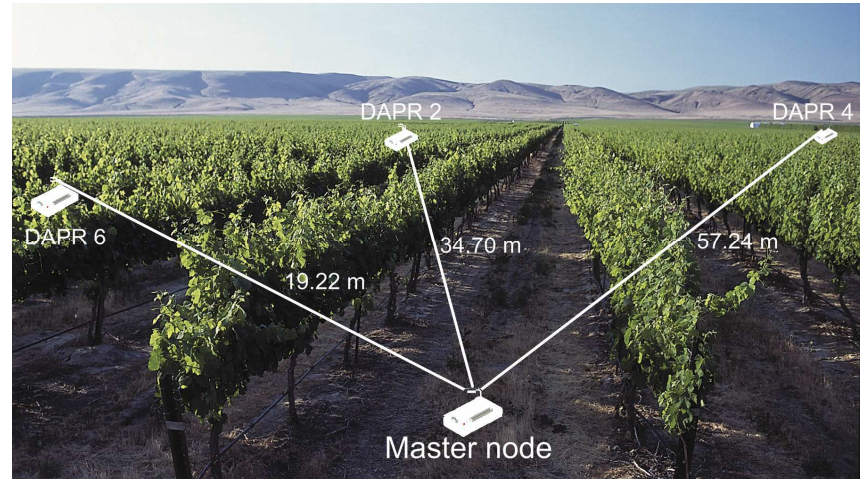

Figure 4 - InterNodal Network disposition

\section{Results in Precison Agriculture}

To demonstrate the potentialities of WSNs we present some results from our InterNodal network practical tests in an agricultural facility. The DAPR nodes were placed on the field at a variable distance from each other but the master node was always at a fixed position. By using the developed computer programmes, several tests were made to measure the received power in each DAPR node. The DAPR nodes were deployed on a field with $2 \%$ inclination, Fig. 4.

The distance from the Master node to node 4 is $\sim 58$ meters, while the distance to node 6 it is $\sim 19$ meters, and to node 2 it is $\sim 35$ meters. All the nodes were placed at a height of 1.30 meters from the ground. Table I presents the power received by each DAPR node (2, 4, and 6), and identifies the nodes that provide access to the respective DAPR node from the Master node in four different networks configurations. If it is different from the Master node then there is multi-hop. As an example, it can be seen that for configuration number 2, the multi-hop communication from DAPR node 4 reaches Master node through an initial hop from DAPR node 4 to node 2 , followed by a connection from node 2 to node 6 , and a final hop from node 6 to the Master node.

Table I - InterNodal Network results

\begin{tabular}{ccc}
\hline \multirow{2}{*}{$\begin{array}{c}\text { Configuration } \\
\text { number }\end{array}$} & \multicolumn{2}{c}{ DAPR 2 } \\
\cline { 2 - 3 } & $\begin{array}{c}\text { Received } \\
\text { power [dBm] }\end{array}$ & $\begin{array}{c}\text { Node that } \\
\text { Provides access }\end{array}$ \\
\hline 1 & 93 & Master node \\
2 & 100 & DAPR 6 \\
3 & 110 & Master node \\
4 & 105 & DAPR 6 \\
\hline \multirow{2}{*}{$\begin{array}{c}\text { Configuration } \\
\text { number }\end{array}$} & Received & DAPR 4 \\
\cline { 2 - 3 } & power [dBm] & Provides access \\
\hline 1 & 104 & DAPR 2 \\
2 & 111 & DAPR 2 \\
3 & 104 & DAPR 2 \\
4 & \multicolumn{2}{c}{ DAPR 6 6 } \\
\hline \multirow{2}{*}{ Configuration } & Received & Node that \\
number & power [dBm] & Provides access \\
\hline 1 & 103 & Master node \\
2 & 101 & Master node \\
3 & 101 & DAPR 2 \\
4 & 100 & Master node \\
\hline
\end{tabular}


Measurements of the received power were performed in each DAPR node for each deployment configuration. From a given configuration to the next one, a reset operation was performed, cleaning all the connections between nodes (DAPR nodes and Master node). By analyzing the results, the received power from DAPR node 6 is larger than the one from the remaining DAPR nodes, which is according to our expectations, since as the distance between each DAPR node and the Master node increases, the received power decreases.

\section{Summary and conclusions}

This Section addressed the conception of an entire WSN for precision agriculture monitoring purposes. This WSN is capable of establishing all the network connections in an automatic way, measuring physical values from the surrounding environment in a vineyard, and triggering devices that are attached to sensor nodes. Examples are temperature, $\mathrm{CO}_{2}$ level, and moisture level. The WSN itself (sensor nodes, master node, monitor software, and sensor node firmware) was developed, and all protocols used in data exchange communications were developed from the scratch.

All the tests of the automatic configurations of the experimental InterNodal network in outdoor and indoor scenarios presented a $91 \%$ of success meaning that, in ten attempts to configure the network only one attempt of automatic configuration was not successful, i.e., the interconnection of all the wireless sensors nodes of the network was not achieved. This event can be justified by the prompt attenuation of the transmitted signal, e.g., owing to obstacles. Another aspect to consider is the possibility of expanding the InterNodal network, and enabling a simple implementation in other operation scenarios of operation.

InterNodal network allows for establishing control points for the sensors (set points) and has the capability to trigger an alarm when an emergency situation appears, e.g., voltage drops in batteries, imperfections of analogue-digital conversion, etc.

\section{WEARABLE APPLICATIONS}

This Section addresses the integration of WSNs technologies in fabrics (wearable devices). The concept behind this is very simple: create a device that a patient could easily wear and that could monitor his vital signs by sending an alert if something abnormal is detected. Furthermore, these devices could be used for:

- Monitoring patients inside the hospital without the need of a dedicated nurse;

- Allowing patients to have their recovery (from surgery, disease, etc) at home while still being monitored;

- Monitoring patients that live far from Hospitals;

- Prematurely detecting diseases on healthy persons, e.g., sudden infant death syndrome (SIDS);

- Detecting casualties in the battle field (military) or on streets (police);

- Monitoring athletes activity;

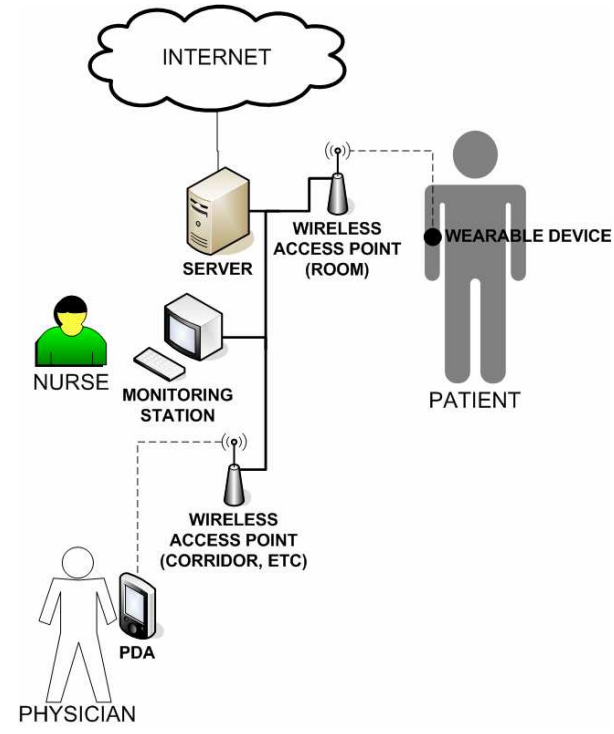

Figure 5 - Wearable technology being used within a Hospital

An example of this type of scenarios can be found in Fig. 5. The patient is being monitored by a device that wirelessly communicates with the hospital network. A server is used to record the information of the patient, and to allow for accessing it from the outside, e.g., by a specialist. The patient can be either in a hospital or at home, and his vital signs are being sent to the Hospital. A nurse is monitoring the patient (better saying, he/she, could be monitoring the entire patients on that area of the Hospital) on his/her office, waiting for any signal of alert. A physician that is going to visit the patient uses his/her PDA to access the historical and recent changes on the patient condition, and can also be warned of an emergency situation on another patient in real-time.

The use of a wearable device will allow for this monitoring process to be continuous no matter the location of the patient (in his bed, while moving in the corridor, in the toilet, at home, in the ambulance, etc). Since one of the requirements is individual mobility, the use of WSN is one of the best solutions for wearable devices. Remember that the device must communicate in real time with the monitoring station while the patient/user is moving, what would be impossible if a wired solution was used. Although a wired solution can be used to connect the sensors, for practical reasons WSNs suit better; otherwise the patient in Fig. 6 would be covered by wires to interconnect all the sensors. The use of WSN to form a BAN (Body Area Network) and to communicate to the outside poses challenges similar to the ones related to other applications. First, the aspect of the autonomy of the network: each node is limited to the power offered by its battery, and it is desirable that it lasts as much as possible. A power management scheme is required, and it is required not only that it aims to save energy but also that it acts as soon as possible in the occurrence of an emergency. Varshney and Sneha [3] suggested a few protocols based on the signals being monitored. If everything is normal a low-power consumption protocol is used. However, if an emergency is detected the system uses all its power to send the alert. 


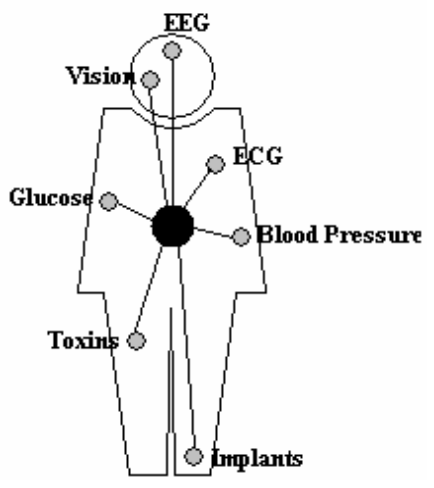

Figure 6 - A Body Area Network (BAN)

Second, we have the security and privacy issue: by sending information over a wireless connection everyone in the range of the signal can capture/destroy/manipulate that information. An eavesdropper can get sensitive data and do whatever he wants with it. The use of cryptography and/or authentication messages is very limited by the resource-constraints of the sensor nodes. The power, memory and computational capability of each node are very limited, and therefore the cryptographic algorithms must be very "light weighted". Therefore the communications must be reduced to the minimum. More complex applications of WSNs to wearable technologies include the monitoring of sports teams activity.

\section{CONCLUSIONS}

WSNs have a vast range of application, including automotive, wearable and agriculture fields. From these fields of application, nowadays, the first one is the less developed one, with only a few solutions implemented, e.g., tire pressure measurement with a $2.4 \mathrm{GHz}$ Zigbee compatible mote. In the area of precision agriculture applications, WSNs allow to monitor the local weather conditions and take decisions based on that information, such as watering a crop only when it is needed, avoiding water wasting. This also allows for optimising the production. As in the wearable field, WSNs are revolutionizing the way healthcare is being provided to people, it allows for some freedom without compromising the treatment. Sportsmen, coaches and their teams can also benefit from these technologies, reaching better performances.

In practical terms, WSNs were used to replace some wired sensors used in a car, or to allow for collecting of data (identification of incorrect tire pressure, verification of illumination, and evaluation of the vital signals of the driver) that otherwise would imply a complicated solution. We were able to successfully collect information from the vineyard environment and record it into the database by using either single-hop or multi-hop communications. Examples are temperature, $\mathrm{CO}_{2}$ level, and humidity level. The research perspectives on WSNs applied to wearable technologies constitute a challenge, and wireless solutions for BANs in the context of fabrics are being addressed for healthcare applications.

A killer cocktail of applications is being sought, and is really needed to enable mass production of small nodes with reduced size and cost, while increasing WSNs overall performance.

\section{ACKNOWLEDGMENT}

This work was supported by UDR (Unidade de Detecção Remota), Department of Physics from University of Beira Interior and also was partially funded by MobileMAN (an internal project from Instituto de Telecomunicações), by IST-UNITE, and by "Projecto de Re-equipamento Científico" REEQ/ 1201/EEI/ 2005 (a FCT Project).

\section{REFERENCES}

[1] Mainwaring A., Polastre J., Szewczyk R., Culler D. and Anderson J. "Wireless Sensor Networks for Habitat Monitoring", in Proc. of the 1st ACM international workshop on Wireless sensor networks and applications, Atlanta, Georgia, USA, 2002.

[2] Yu L., Wang N. and Meng X., "Real-time Forest Fire Detection with Wireless Sensor Networks", Wireless Communications, Networking and Mobile Computing, 2005.

[3] Varshney U. and Sneha S., "Patient Monitoring Using Ad Hoc Wireless Networks: reliability and Power Management", IEEE Communications Magazine, Apr. 2006, pp. 49-55.

[4] Winters J.M., Wang W. and Winters J.M., "Wearable Sensors and Telerehabilitation", IEEE Engineering in Medicine and Biology Magazine, May/June 2003, pp. 56-65.

[5] http://www.nhtsa.dot.gov/staticfiles/DOT/NHTSA/Rulemaking/Rules/As sociated\%20Files/TPMS-2005-FMVSS-No138.pdf, 8/2/2007.

[6] http://www.sensatex.com/, 18/1/2007.

[7] Noury N., Dittmar A., Corroy C., Baghai R., Weber J. L., Blanc D., Klefstat F., Blinovska A., Vaysse S. and Comet B., "VTAMN - A Smart Clothe for Ambulatory Remote Monitoring of Physiological Parameters and Activity", in Proc. of the $26^{\text {th }}$ Annual International Conference of the IEEE EMBS, San Francisco, California, USA September 2004.

[8] Bourdon L., Coli S., Loriga G., Taccini N., Gros B., Gemignani A., Cianflone D., Chapotot F, Dittmar A. and Paradiso R., "First Results with the Wealthy Garment ElectroCardiogram Monitoring System", Computers in Cardiology, 2005, pp. 615-618.

[9] http://www.proetex.org/index.htm, 29/1/2007.

[10]http://www.signalife.com/Pages/NewsPDF/2006/PR08282006.pdf, $15 / 3 / 2007$.

[11]http://www.ist-ubisecsens.org/wp4.php , 1/2/2007.

[12]Baggio A., "Wireless sensor networks in precision agriculture" in Workshop on Real-World Wireless Sensor Networks, Stockholm, Sweden, June 20-21, 2005.

[13]http://wireless.industrial-networking,com/articles/articleprint.asp ?id=837, 7/2/2007

[14]Crossbow Solutions, "Motes in Controlled Environment Agriculture", from Fourth Quarter, volume 8, 2005.

[15]Tavares J., Velez F. J. and Ferro J., "Application of Wireless Sensor Networks to the Automobile", in Proc. of ConfTele' $2007-6^{\text {th }}$ Conference on Telecommunications, Peniche, Portugal, May 2007.

[16]Luz G., "Roteamento em Redes de Sensores", Instituto de Matemática e Estatística, Universidade de São Paulo, Brasil, Nov. 2004 (http://grenoble.ime.usp .br/movel/roteamentosensores.pdf).

[17] http://internodal.no.sapo.pt, 28/2/2007.

[18] Borges L., Carvalho F., Lebres A., "InterNodal Network - An Autoconfigurable Wireless Network", in Proc. of Conftele' $2007-6^{\text {th }}$ Conference of Telecommunications, Peniche, Portugal, May 2007. 